\title{
A INOVAÇÃO E SEUS IMPACTOS NO CONTEXTO DA CULTURA EDUCACIONAL
}

COELHO, Juliane Maria Pereira ${ }^{1}$

COLVARA, Jonas.Santos ${ }^{2}$

ROLIM, Sandra Catarina ${ }^{3}$

COELHO, Juliane.Maria.Pereira; COLVARA, Jonas.Santos; ROLIM, Sandra Catarina. A Inovação e seus Impactos no Contexto da Cultura Educacional. Revista Científica Multidisciplinar Núcleo do Conhecimento. Edição 04. Ano 02, Vol. 01. pp 158-178, Julho de 2017. ISSN:2448-0959

\section{RESUMO}

Este é um trabalho desenvolvido para a disciplina de Culturas, Saberes e Práticas. Através de pesquisas bibliográficas buscam-se respostas para as indagações levantadas pelas leituras realizadas sobre o tema: A Inovação e seus impactos no contexto da cultura educacional. Indagações essas como por exemplo: $O$ que é cultura? O que é inovar? Por que inovar? Quais os tipos de inovação? Quais os impactos das inovações na cultura educacional? As pesquisas bibliográficas realizadas mostraram-se muito enriquecedoras nas elucidações a essas indagações ressaltando os principais conceitos. Através das definições recolhidas nos dicionários começamos a trilhar os estudos que nos levarão ao conhecimento advindo da pesquisa sobre o tema da Inovação e seus impactos no contexto educacional. Ao final desse trabalho vamos expor algumas observações acerca de ações inovadoras

\footnotetext{
${ }^{1}$ Mestranda em Ciências da Educação na Universidad e la Empresa- Uruguai.

${ }^{2}$ Mestrando em Ciências da Educação na Universidad e la Empresa- Uruguai.

${ }^{3}$ Mestranda em Ciências da Educação na Universidad e la Empresa- Uruguai.
} 
propostas numa escola de formação técnica / profissionalizante nas áreas de enfermagem e radiologia e seus principais impactos, positivos e / ou negativos.

Palavras-Chaves: Inovação, Cultura, Educação.

\section{INTRODUÇÃO}

Esse é um estudo em que se procura, através da pesquisa no referencial bibliográfico, o entendimento sobre como ações inovadoras podem impactar na cultura educacional. Buscam-se respostas para questões primeiramente básicas tais como esclarecer o que é inovação, o que é cultura, o que é educação e porquê inovar.

Ao final espera-se reconhecer quais os impactos que essas ações inovadoras podem atribuir positiva ou negativamente na cultura dos processos educacionais.

Temos a ousada intenção de nortear as possíveis inovações no contexto da cultura educacional através da análise das leituras de referenciais bibliográficos e expor a aplicação das inovações pedagógicas e educacionais em uma escola de formação técnica / profissionalizante nos cursos de enfermagem e radiologia e os principais impactos dessas ações inovadoras no âmbito administrativo, pedagógico e entre o corpo docente e discente.

\section{DESENVOLVIMENTO}

Inicialmente devemos analisar o conceito de palavras introduzidas nesse trabalho com o intuito de esclarecer não só semanticamente, mas o seu significado aplicado às ações.

\subsection{O QUE É CULTURA?}

Ressaltando os conceitos contidos nos dicionários temos que cultura é: "Ação, efeito, arte, ou maneira de cultivar. Constituição de conhecimentos básicos indispensáveis para o entendimento de quaisquer ramos do saber humano. (DICIONÁRIO HOUAISS, 2004) 
Ainda encontramos: Conjunto de hábitos sociais e religiosos, das manifestações intelectuais e artísticas; normas de comportamento, saberes e hábitos que diferenciam um grupo de outro grupo. (DICIONÁRIO ONLINE DE PORTUGUÊS)

Devemos buscar esses conceitos de cultura para que possamos destacar sua importância nas discussões atuais quando pretendemos aliar esse conceito às questões de ações inovadoras.

A contemporaneidade da definição aplica-se partindo do princípio que são esses conceitos que dão sentido às ações humanas, pessoais ou de grupos que buscam maneiras de demonstrar seus hábitos, suas expressões e maneiras como se comunicam, dando dessa forma significado às suas ações.

Segundo Hall (1997); a cultura sempre foi importante nas ciências humanas e sociais, pois os estudos das linguagens, da literatura, das artes, sempre fundamentaram o tema, embora não fosse trivial o entendimento de que esses estudos compusessem um conjunto diferenciado de significados, ou seja, uma cultura, na concepção desse autor.

Percebemos assim que o conceito de cultura determina e é importante para os significados, para o melhor entendimento do indivíduo e de seu grupo. A cultura assume um destaque especial para pensar o mundo, suas comunidades, e seus indivíduos.

Já para Williams (2008); os sentidos da cultura serviram como resultado de forma precursora de convergência de interesses, das quais se destacam duas principais, frequentemente classificadas como idealista (ênfase no espírito formador) e materialista (com ênfase numa ordem social global), que contribuem para o estudo das relações entre as atividades culturais e as demais formas de vida social.

Nossa sociedade, notadamente multicultural, necessita de arranjos para que se busque uma convivência que nos dê uma empatia de troca entre conhecimentos pertinentes a determinado grupo e que possam também influenciar coletivamente e 
positivamente a formação de um pensamento global, apesar das diferenças culturais características.

Segundo Machado, (2014), a cultura possui vários conceitos advindos das ciências sociais e humanas. O repasse da cultura aos novos integrantes e à própria sociedade se dá por meio da busca da objetivação ou concretização daquilo que é subjetivo.

Esse desempenho na construção através da cultura, que surge em praticamente todos os aspectos de seu papel social devem ser reconhecidos como algo destacável uma vez que a cultura surge como elemento primordial na organização da sociedade bem como na construção de seus atores sociais. Deparamo-nos com uma real revolução cultural abrangente em todos os seus aspectos.

Chegamos ao ponto onde vislumbramos a cultura globalizada. Essa globalização cultural afeta sobremaneira a sociedade mundial quanto a posicionamentos políticos, suas formas de competitividade econômica e talvez, fundamentalmente, suas formas de comunicação.

Um mercado global é criado a partir do momento que sua homogeneidade cultural está crescendo de tal maneira que está tornando inevitável o encurtamento das distâncias ideológicas entre nações e sendo assim está dando início a processos políticos econômicos altamente eficazes para esses governos. (Dale, 2004)

Mudanças que ocorrem em todas as esferas sociais e culturais, e que visam principalmente a percepção da importância da cultura para se pensar esse novo mundo, atingiram diretamente um componente essencial desse olhar: a cultura da educação.

Entendemos então que, a educação possui como objetivo o desenvolvimento de uma consciência cultural de emancipação. Essa liberdade é necessária para a formação de sujeitos críticos que se apresentam como atores sociais diante da história, da cultura e da natureza. 
Senão da maneira libertadora a cultura da educação tende a levar uma sociedade totalitária a manifestar-se de forma a propagar a ideia de dependência, da servidão. Nesse aspecto, é importante a análise feita por Santiago, (2005), onde ele afirma que a educação perderá sem dúvida sua utopia e ingenuamente se quiser trombar de frente com o mundo burocratizado, mas sempre terá uma chance caso volte-se para o indivíduo. Dificilmente o ensino reverterá os passos da civilização que em simultâneo progride e regride, mas sempre poderá combater no indivíduo a suscetibilidade a essa situação.

Levando as concepções acima descritas para a visão da cultura na educação, temos segundo Julia, (2012); que esse aspecto da cultura pode ser descrito como um conjunto de normas que definem conhecimentos a ensinar e condutas a inculcar, e um conjunto de práticas que permitem a transmissão desses conhecimentos e a incorporação desses comportamentos.

\subsection{O QUE É EDUCAÇÃO}

Ato ou efeito de educar; aperfeiçoamento das faculdades físicas intelectuais e morais do ser humano; instrução e ensino. Civilidade. (DICIONÁRIO HOUAISS 2004)

Ação ou efeito de educar, de aperfeiçoar as capacidades intelectuais e morais; capacitação e/ou formação das novas gerações de acordo com os ideais culturais de cada povo. (DICIONÁRIO ONLINE DE PORTUGUÊS)

Segundo o RELATÓRIO PARA A UNESCO DA COMISSÃO INTERNACIONAL PARA A EDUCAÇÃO DO SÉCULO XXI, Delors, (2010), a educação deve enfrentar um problema porque, ela se situa, mais do que nunca, no âmago do desenvolvimento da pessoa e das comunidades; sua missão consiste em permitir que todos, sem exceção, façam frutificar seus talentos e suas potencialidades criativas, o que implica, por parte de cada um, a capacidade de assumir sua própria responsabilidade e de realizar seu projeto pessoal. Ela deve permitir que cada um venha a tomar consciência de si próprio e de seu meio ambiente, sem deixar de desempenhar sua função na atividade profissional e nas estruturas sociais. 
Não é possível falar e pensar em educação separadamente do homem. Haveremos de pensar o homem como sujeito do processo educacional. O processo da formação da educação humana envolve o indivíduo, mas não o separa do seu coletivo.

Dessa forma só podemos mesmo pensar na educação como uma ação libertadora, carregada de um sentido de autoconhecimento e consequentemente da construção de seres humanos ativos, críticos e que exercem sua função cidadã nessa sociedade do conhecimento.

Devemos compreender a essência da educação para nos dirigirmos a suas características específicas, sua natureza permeia conhecimentos, ideias, valores, simbologias, atitudes, mas com todo seu valor voltado para o interior do homem.

Como ressalta Saviani, (2015), a educação não se reduz ao ensino e este, sendo um aspecto da educação, participa da natureza própria do fenômeno educativo.

Sistemas educacionais que pensam somente no conhecimento a despeito de outras práticas de aprendizagem estão fadados ao insucesso, pois é imprescindível entender a educação como um todo e para um todo. Através dessa ideia é que a expectativa para o futuro seja no sentido da inspiração e da orientação quanto a reformas educacionais, bem como a elaboração de programas ou ainda a definição de novas políticas pedagógicas.

E quais as perspectivas para a educação num momento que vivemos?

A ideia de perspectivas vem completa do pensamento de novidades, um projeto, novos ideais. Possibilidades.

Segundo Gadotti, (2000) muitos educadores, perplexos diante das rápidas mudanças na sociedade, na tecnologia e na economia, perguntam-se sobre o futuro de educação, alguns com medo de perder sua posição por não saber o que fazer.

A partir das reflexões sobre a nova educação e suas perspectivas, seu futuro, nasce um pensamento de mudanças, alinhavado pelo fenômeno da globalização que deu 
novo ânimo as inovações com o objetivo de se buscar uma educação igualitária no sentido da formação do humano cidadão, consciente, livre e aberto às possibilidades do alcance do conhecimento. Vem aí a ideia ou o ideal da inovação.

Incorporado aos conceitos descritos acima, passemos a outro tema: A Inovação.

\subsection{O QUE É INOVAÇÃO?}

Semanticamente a Inovação é definida como:

Ato ou efeito de inovar, renovação. (DICIONÁRIO HOUAISS,2004)

Novidade, aquilo que é novo. Qualquer modificação ou ação de fato ou de direito que desperta ou incita o interesse em diversas áreas. (DICIONÁRIO ONLINE DE PORTUGUÊS).

Isso leva a questões de que inovação é todo processo que nos traz uma ideia de ação que transforma o já existente, aquilo que busca introduzir algo de novo em rotinas já desgastadas, mas que ainda possuem em sua essência o espírito do engrandecimento do indivíduo ou de seu grupo.

Segundo Garcia, (2013); do latim "innovare", fazer inovações, produzir algo novo. A resposta parece simples: em um mundo no qual as mudanças ocorrem de forma veloz e intensa, questionando velhos modelos, não há quem não se preocupe em buscar adaptar-se ao novo.

Com certeza a inovação tem sido um objetivo comum a diferentes tipos de organizações, evidentemente que cada uma delas com suas próprias características e finalidades, assim em cada realidade vários aspectos devem ser postos a fim de agregar ou eliminar barreiras que possam dificultar a implementação de ações inovadoras.

Estudos sobre inovações buscam inicialmente avaliar e demonstrar a viabilidade do ambiente dessa ou aquela organização em receber essas ações. Segundo Dobni, 
(2008); esses estudos buscam identificar aspectos culturais engendrados nesse processo.

Em nossa moderna sociedade do conhecimento, o novo torna-se sempre algo com uma sombra temerária, em desafio para as organizações e seus atores, no sentido de que pelo lado das organizações, essas, precisam manter-se atualizadas e competitivas, enquanto que pelo lado dos indivíduos o temor de serem ou não capazes de manterem-se atualizados, criativos numa organização que os engolem em um passo em falso.

Segundo Sergio, (2015); o processo inovador caracteriza-se como sendo um processo que necessita ser gerenciado e estar alinhado às perspectivas da organização. A inovação é fundamental para as organizações, de modo que proporcione produtos novos ou melhores aos seus clientes.

Novas ideias correspondem à matéria-prima para a inovação. No entanto é necessário analisar alguns aspectos de cada nova ideia, pois esse estudo vai impactar diretamente no fracasso ou sucesso dos processos inovadores.

Du Preez, Louw e Esmann, (2006); salientam que, para ocorrer o processo de criação de novos conhecimentos, é necessária a interação entre indivíduos com diferentes conhecimentos. Contudo, é extremamente importante a construção e a manutenção do ambiente organizacional favorável à inovação, pois, só assim, efetivamente, a gestão da inovação obterá êxito.

Sendo assim entende-se que a interação entre as pessoas favorece o processo de inovação e consequentemente o processo de criação de ideias. A geração de ideias possui valor real para as organizações, pois pode representar o ponto inicial do processo de inovação e auxiliar na identificação de oportunidades.

Atualmente no Brasil, os resultados das inovações educacionais para uma prática pedagógica que se coloque à altura dos desafios educativos apresentados, não traz muito otimismo para uma análise mais acurada do processo de escolarização. Esse quadro poderá levar a " opção do derrotismo e da amargura. Em oposição a ela, está 
a alternativa de ir ao encontro das transformações sociais e corporar-se ao processo de mudança" (Marchesi e Martin, 2003).

De acordo com Cardoso, (1997) a inovação é, pois, uma das exigências prioritárias do presente se atendermos à necessária participação do homem na construção das sociedades contemporâneas. A sua pertinência e necessidade são, hoje, largamente aceites.

$\mathrm{Na}$ análise das inovações educacionais no Brasil, no estudo de Ferretti, (1980), ele afirma que devido a uma série de condições adversas ao exercício profissional, os professores tendem a recusar os procedimentos inovadores

Deparamo-nos com uma das principais dificuldades à assimilação das atitudes inovadoras que é a resistência dos professores em relação as novas práticas propostas. As necessidades sociais de melhorias e modernização das ações em prol da modernização exige uma opção dos profissionais em relação à seu papel dentro da estrutura organizacional. Seus atores podem optar pela mudança de postura e ações, ou não. Se a opção do professor, nesse caso, for a última suas motivações, ações e métodos orientarão todo o processo no sentido de não desenvolver essas ações inovadoras e suas transformações. Caso a pessoa tenha a consciência da importância desse movimento de inovação social e organizacional sua determinação exigirá esforços para que possam se adaptar às novas condições de sua organização.

Olson e Bruner, (2000), apontam que "nossas interações como outros são profundamente afetadas por nossas teorias intuitivas cotidianas sobre como nossas próprias mentes e as mentes dos outros funcionam". Os professores não são guiados apenas por essas teorias, denominadas "psicologia popular"; nas suas práticas pedagógicas são guiados, também, pela "pedagogia popular", que constitui um conjunto de suposições sobre como as pessoas aprendem. Assim, "quaisquer inovações terão de competir com as teorias populares, substituí-las ou, de outra maneira, modificar essas teorias que já guiam tanto os professores quanto os alunos". 
É aqui que a questão de escolha surge ao nível das pessoas. A visão é que uma educação transformadora proveniente de ações inovadoras se faz com pessoas; professores, alunos, gestores, pais, comunidade. É uma ação no agir cotidiano das pessoas e das organizações.

Onde demonstra-se a interligação entre a cultura e a inovação?

Alguns autores ressaltam que a cultura pose se constituir como uma barreira à inovação, como observam Kaasa e Vadi, (2010); quando registram o fato de que a cultura tanto pode unificar comportamentos e pessoas como também pode constituirse em barreiras entre as mesmas. Os autores acrescentam que "cultura afeta indivíduos, ações coletivas e entendimento, comportamentos em termos de risco.

\subsection{POR QUE INOVAR?}

A inovação é o princípio chave da capacidade de desenvolvimento e da competitividade que as organizações precisam adquirir para sair do estado mediano a que chegou se quiser manter-se atualizada e ainda no ambiente competitivo.

A inovação auxilia também no posicionamento da organização nas estratégias de segmentação no mercado. Nessa perspectiva, Barbieri, (2003); argumenta que organizações são reconhecidas pelo mercado por sua capacidade de inovação.

A inovação na educação veio do âmbito das organizações de produção e administração. Segundo Messina, (2001), nas décadas de 1950/60, os teóricos da inovação concebiam-na como um processo em etapas previsíveis, desde a gestação das ideias até a implementação e generalização.

$\mathrm{Na}$ grande maioria dos conceitos de inovação em educação percebemos uma recorrente assertiva nas relações de que essas ações têm relação com mudanças na mediação pedagógica através da colocação de novos recursos, novos materiais, novas posturas, visando o novo objetivo colocado. 
O conceito de inovação para Teixeira, (2010); compreende necessariamente a introdução de 6 uma novidade num sistema educativo que promova uma real mudança resultante do esforço deliberado e conscientemente assumido, fruto de uma ação persistente e integrada num processo dinâmico, que objetive uma melhoria pedagógica.

Uma conceituação interessante para a inovação em educação nos é apresentada por Saviani, (1995); quando nos fala que inovar, em seu sentido próprio, será colocar a educação a serviço de novas finalidades, vale dizer, a serviço da mudança estrutural da sociedade. Levantam-se questões sobre o papel da educação no processo da mudança social.

Diante do apresentado até o momento devemos considerar que as ações de inovação na educação como uma busca de alcançar novas respostas para os desafios de capacitar e adequar novos sistemas educativos à moderna sociedade de conhecimento devem ser verificadas avaliativamente pelas organizações educativas de acordo com as reais contribuições que tais inovações podem oferecer a melhoria do processo educacional produzindo os benefícios esperados, investigando não somente a inserção, mas sua apropriação das novas ações entre todos os envolvidos; mudando a visão de que serão instrumentos restrito para instrumentos de ferramentas de ensino, para potencializar os processos de aprendizagem e enfatizar não somente o impacto cultural, mas sua condição de produto cultural e social.

Racionalizarmos a cultura da inovação à partir das palavras de Dobni, (2008): a cultura de inovação associa-se aos impactos no desempenho organizacional, sendo considerada como elemento vital para que ela ocorra, especialmente por prover vantagem competitiva.

Entende-se a esse ponto que a influência das inovações nas questões culturais em seus demasiados ramos demonstra, segundo Mclean, (2005), que a cultura de inovação faz parte da cultura maior de uma instituição, com o objetivo de seu desenvolvimento e aprimoramento nos modernos conceitos de evolução organizacional. 


\section{OS IMPACTOS DE AÇÕES INOVADORAS NUMA ESCOLA DE FORMAÇÃO TÉCNICA PROFISSIONALIZANTE}

Por Mendonça, (2014), toda expansão do ensino técnico de nível médio vem sendo declarada pelo governo brasileiro como intenção de democratizar o acesso, aumentando o número de vagas, porém não temos nos documentos oficiais políticas que apontem como esta formação está sendo ofertada quanto à qualidade e a real contribuição desta formação na vida dos alunos egressos destes cursos.

$\mathrm{Na}$ escola profissionalizante em questão, localizada na cidade de Belo Horizonte, Minas Gerais, Brasil, temos uma atividade educacional que monta de (2004), quando seu foco era e ainda é a formação técnica na área da enfermagem e da radiologia.

O desenvolvimento da escola foi notável quando os governos estadual e federal incentivaram bolsas de estudo para pessoas interessadas no ingresso mais rápido no mercado de trabalho, via projetos educacionais conhecidos como PEP (Programa Estadual de Profissionalização) e o PRONATEC (Programa Nacional de Formação Técnica). Adultos jovens e adultos da terceira idade veem aí uma oportunidade de adentrar ou voltar ao mercado de trabalho.

Essa escola profissionalizante teve seu momento de declínio quando as políticas econômicas e educacionais do governo começaram a ruir e o pagamento das bolsas de estudo ofertadas a esses alunos passam a não acontecer. Acabaram as vagas originárias desses programas restando somente os alunos que já estavam matriculados, causando um déficit financeiro considerável à instituição e uma grande incerteza aos alunos quanto ao término de seus cursos.

Nas últimas décadas, a preocupação com a disseminação e a democratização do acesso à educação para atender a grande massa de educandos, evidenciou a importância da educação a distância. $\mathrm{O}$ advento das tecnologias de informação e comunicação trouxe novas perspectivas para a educação a distância, levando universidades, escolas, centros de ensino, organizações empresariais e grupos de profissionais de educação, a se dedicarem ao desenvolvimento de cursos a distância 
com suporte em ambientes digitais de aprendizagem acessados via internet, os quais assumem distintas abordagens. Almeida, (2011).

A partir da necessidade de uma modernização e também uma diminuição de custos a escola profissionalizante adotou em 2015 algumas disciplinas na modalidade online para os novos alunos. Não só disciplinas, bem como suas avaliações de desempenho passaram a ser realizadas num ambiente virtual de aprendizagem (AVA) através da plataforma Moodle.

A EaD (Educação a Distância) consiste então, em um processo que enfatiza a construção e a socialização do conhecimento, de forma que qualquer pessoa, independentemente do tempo e do espaço, possa tornar-se agente de sua aprendizagem, devido ao uso de materiais diferenciados e meios de comunicação que permitam a interatividade, a interação (síncrona ou assíncrona) e o trabalho colaborativo/cooperativo.

Descobre-se nesse momento a dificuldade de implementar verdadeiramente esse novo projeto de gestão educacional. Dificuldades como a aceitação dos alunos frente a novidade do projeto pedagógico da escola, uma certa resistência dos professores quanto à perspectiva de terem que se atualizar com a nova modalidade de ensino /aprendizagem, e até a falta de um planejamento quanto a implantação de novos computadores tornam-se variáveis muito expressivas.

Para entendermos o organograma da escola, a instituição é hierarquicamente formada por uma diretoria, gerência financeira, gerência de negócios, coordenação pedagógica e de cursos e o corpo docente.

No início do ano de 2015 surgem as propostas pelo Conselho Acadêmico (diretoria, administração, setor pedagógico, e docentes) de ações para implementar essa nova modalidade de ensino à distância, dentro do modelo de gestão educacional vigente para modernizar o projeto pedagógico educacional.

Ao longo dos meses em que essas ações se apresentam os indicadores de melhorias dos processos da inovação educacional (EAD) não se sobressaem, uma vez que se 
percebe uma imobilidade da direção da escola em empenhar-se na implementação desses novos valores.

Não observamos ainda uma relação saudável entre a coordenação pedagógica e o corpo docente onde existe uma cobrança na atuação desses atores sem proporcionarIhes um devido treinamento ou considerando suas reivindicações, e que ainda sofre pela postura de inércia da instituição frente a esse novo desafio da implantação da modalidade da Educação à Distância.

Outro ponto a ser levantado é a falta de investimento na infraestrutura oferecida pela a instituição para que o ambiente virtual de aprendizagem venha a funcionar a contento, trazendo assim benefícios já conhecidos da Educação à Distância. Como uma cultura de inovação, a construção do conhecimento do ser humano, pode aprimorar-se dessa forma?

Em relação ainda as inovações vejamos a posição dos professores. A escola em que tratamos nos traz um corpo docente bastante especializado e com um alto nível de comprometimento com a cultura de ações inovadoras como a implantação do sistema de Educação à Distância. Apesar de poucos terem conhecimento prático existem um grande senso profissional para que essa dificuldade seja ultrapassada, na forma de treinamentos eles buscam orientação para sua prática.

Segundo Moran, (2015), professores e coordenadores sofrem o impacto inesperado dos bons cursos de educação a distância. Na implantação da EAD, costuma haver, uma certa desconfiança e até um distanciamento generalizado.

Nota-se ainda a existência de uma dificuldade na relação professor / aluno. Surgem as figuras dos professores conteudistas e os professores tutores das disciplinas. Essa relação com um professor tutor torna-se diferente das relações habituais. Tem-se, a partir da aplicação dos meios da EAD um novo conceito de tutoria que vai exigir uma relação mais constante, com um contato mais próximo para que seja realmente eficaz.

Em relação aos alunos nem todos conseguem manter uma disciplina de estudos e de contato com os professores tutores assumindo assim a responsabilidade de um 
aprendizado talvez deficitário, no momento em que esses alunos tornam 0 aprendizado como uma atividade individualista, em que ele não busca o apoio ou a intervenção desse tutor na formação de seu conhecimento, mesmo com o incentivo e disponibilidade de ferramentas oferecidas pela instituição.

Na maioria das escolas, as atividades a distância não implicam até agora em nenhuma diminuição das aulas presenciais. Duplica-se, na prática, o tempo de atendimento, as atividades de professores e alunos. Moran, (2015).

Para o aprendizado de qualquer disciplina é preciso que seus objetivos estejam bastante claros. Assim o currículo adotado pela escola deve descrever com precisão como isso será feito e esse é outro impacto que se observa na vivência da implantação da EaD.

Objetivamente a evolução desse processo não está bem descrita no planejamento pedagógico da instituição fazendo com que uma série de equívocos ocorram no desenvolvimento do projeto, não só no processo da infraestrutura (aquisição de computadores, bom projeto de instalações para o laboratórios de informática, etc) bem como os processos e procedimentos que deveriam ser instaurados desde as áreas administrativas, passando pelos processos pedagógicos, coordenadores, corpo docente e finalmente o corpo discente.

As disciplinas escolhidas para serem realizadas online causam certo desconforto nos alunos uma vez que por se tratar de cursos na área da saúde, matérias como Anatomia e Fisiologia Humana são de extrema importância em sua formação e passaram à essa modalidade. No entendimento desses alunos e mesmo de alguns professores essa é uma disciplina que depende muito de uma interação professor/aluno devido às suas características tantos conceituais quanto visuais. Enquanto outras disciplinas completamente teóricas em sua essência ficaram na forma presencial.

As ações inovadoras no sentido da cultura educacional com o objetivo de trazer novos modelos de aprendizagem , modernidade e agilidade na construção do conhecimento 
estão se diluindo no processo, uma vez que a instituição apresenta falhas na implementação dessas inovações, como a escolha equivocada das disciplinas a serem colocadas na modalidade a distância, bem como pela falta de infraestrutura quando não dispõe de um número compatível de computadores para serem utilizados pelos alunos para o desenvolvimento de suas atividades principalmente as avaliações que deveriam ser realizadas nos laboratórios de informática, desacreditando dessa forma, alunos e professores, quanto à eficácia dessas ações inovadoras.

Em um de seus estudos, Bates, (2001), relata que entre os aspectos positivos da inovação da educação a distância existe o fato de que esse modo difunde inovações, instila uma nova cultura, releva e desenvolve talentos etc. Mas aponta também para o amadorismo que geralmente acompanha tais iniciativas, com reflexo na qualidade ou acabamento dos materiais, no fato de só serem difundidos os resultados exitosos (daí abrindo caminho para a repetição de erros), para a quantidade de tempo que os professores gastam no aspecto mais artesanal ou técnico do trabalho, em detrimento daquele propriamente intelectual ou pedagógico.

De acordo com Wentling et al., (2000), a satisfação dos profissionais nas experiências de aprendizagem em ambientes virtuais é importante porque as reações positivas influenciam o apoio organizacional para a constituição de futuras formações e orientam acerca das mudanças nas estratégias formativas atuais. Essa avaliação positiva depende também da interação entre o aprendiz e o ambiente virtual de aprendizagem, expressas pelas características de usabilidade das tecnologias de informação e comunicação e como consequência o sucesso das implantações dessas ações inovadoras.

\section{CONSIDERAÇÕES FINAIS}

A capacidade das instituições de inovar depende de vários fatores, incluindo os aspectos de gestão de recursos humanos e tecnológicos de inovação. A inovação tem sido considerada como essencial para permitir que as instituições e as economias nacionais a prosperar em mercados globalizados e cada vez mais competitivos. 
O grande dilema é que os seres humanos em sua atividade diária são criaturas de hábitos e rotinas, mas a inovação requer o reconhecimento e capacidade de resposta para mudar. Práticas de gestão de pessoas precisam ser criadas e mantidas em um ambiente que apoia a inovação, com a finalidade de motivar e fornecer aos seus personagens as oportunidades para inovar.

A criatividade é a geração de ideias novas e úteis geradas por pessoas, e inovação é a implementação bem-sucedida de tais ideias. Para os estudiosos de culturas de inovação, e ainda mais, da cultura inovadora educacional, a diferenciação entre criatividade e inovação é fundamental, porque é a gestão dessas pessoas, os indivíduos na instituição, que induz a criatividade, ao passo que a inovação - a implementação de ideias criativas opera com o todo.

Independente da forma como o processo de inovação é realizado introduzir uma inovação educativa implica numa mudança planejada com o propósito de dotar a instituição de uma capacidade para satisfazer aos objetivos que motivam a própria inovação. Assim inovação educacional pode ser entendida como a busca de respostas aos desafios presentes na dinâmica aos processos escolares, a partir da análise e reflexão que se faz do contexto sócio - cultural e efetivas contribuições que tais inovações podem oferecer para enfrentar esses desafios.

É preciso considerar mais que os processos racionais da instituição, pois a implementação de um novo comportamento passa pela cultura das organizações.

Para realizar a mudança cultural é necessário que as justificativas do comportamento também sejam mudadas, o que pode significar que as mudanças têm que se basear em razões intrínsecas, em novos valores e crenças.

"A inovação não se decreta. A inovação não se impõe. A inovação não é um produto. É um processo. Uma atitude. É uma maneira de ser e estar na educação" Cardoso, (2003).

Enquanto isso podemos perceber na citada escola que a implantação da modalidade de educação a distância como modelo inovador necessita de ajustes tanto em relação 
aos professores quanto a preparação dos alunos; lembrando ainda das questões estruturais como a presença do material tecnológico necessário e eficiente para o sucesso dessa empreitada.

Temos a conviçcão do caráter inclusivo da modalidade EAD no sentido de democratizar o acesso à educação, mas devemos considerar que as instituições tenham todos os aspectos físicos, educacionais, pedagógicos e tecnológicos de referência para prezar a excelência da qualidade da educação e para que o sucesso na implementação das ações inovadoras dentro da cultura educacional seja exitoso.

\section{REFERÊNCIAS}

ALMEIDA, Maria Elizabeth B. de. Tecnologia e educação a distância: abordagens e contribuições dos ambientes digitais e interativos de aprendizagem. Revista Brasileira de Educação a Distância, p. 6, 2011.

BARBIERI,J.C; Organizações Inovadoras: estudos e casos brasileiros. 2003.

BATES, T. Como gestionar el cambio tecnológico: estratégias para reponsables de centros univesitários: Gedisa Barcelona:, 2001.

CARDOSO, Ana Paula. Educação e inovação. Millenium, 1997.

CARDOSO, A. P. O. A Receptividade à Mudança e à Inovação Pedagógica: o professor e o contexto escolar. Edições Asa. Porto. 2003

DALE, Roger. Globalização e educação: demonstrando a existência de uma "cultura educacional mundial comum" ou localizando uma "agenda globalmente estruturada para a educação". Educação \& sociedade, v. 25, n. 87, p. 423-460, 2004.

DELORS, Jacques et al. Relatório para a UNESCO da Comissão Internacional sobre Educação para o século XXI. Educação: um tesouro a descobrir. São Paulo: UNESCO, 2010. 
DICIONÁRIO ONLINE DE PORTUGUÊS, < http://www.dicio.com.br > acessado em 27 de maio de 2016.

DOBNI, C. B, Measuring innovation culture in organizations: the development of a generalizaded innovation culture construct using exploratory fator analysis. European Journal of Innovation Management, 2008.

DU PREEZ, N.; LOUW, L.; ESSMANN, H. An Innovation Process Model for Improving Innovation Capability. Journal of High Technology Management Research, p. 1-24, 2006

FERRETTI, C. Novas tecnologias, trabalho e educação. Vozes, Petrópolis 1980.

GARCIA, Paulo Roberto Salles. O papel da inovação na educação. Revista de Educação do Cogeime, v.21, n. 40, p. 4, p. 372, 2014

GADOTTI, Moacir. Perspectivas atuais da educação. São Paulo em perspectiva, v. 14, n. 2, p. 03-11, 2000.

HALL, S. A centralidade da cultura: notas sobre as revoluções culturais do nosso tempo. Educação e Realidade, n.2, v.22, p.5, Porto Alegre 1997.

HOUAISS, A. Dicionário da Língua Portuguesa, Objetiva, Rio de Janeiro, 2004

JULIA, Dominique. A cultura escolar como objeto histórico. Revista brasileira de história da educação, v. 1, n. 1 [1], p. 9-43, 2012.

KAASA, A; VADI, M; How does culture contrubuite to innovation? Evidence from European Countries. Economics of Innovation and New Technology, 2010.

MACHADO, Denise Del Prá Netto et al. Cultura de Inovação: elementos da cultura que facilitam a criação de um ambiente inovador. RAl: revista de administração e inovação, v.10, n. 4, p. 164-182, São Paulo , 2014. 
MARCHESI, A. e MARTÍN, E. Qualidade do ensino em tempos de mudança. Artmed Porto Alegre:, 2003.

Mclean, L. D. Organizational culture's influence on creativity and innovation: a review of the literature and implications for human resource development. Advances in Developing Human Resources, (2005).

MENDONÇA, Aline Graciele. Ensino técnico de nível médio: momentos de prestígio e de esquecimento se alternando durante a história da educação profissional no Brasil. Horizontes, v. 32, n. 2, 2014.

MESSINA, G. Mudança e inovação educacional: notas para reflexão. Cadernos de Pesquisa. № 114, 2001, p. 225 - 233

MORAN, José Manuel. Desafios que a educação a distância traz para a presencial. Revista de Ensino, Educação e Ciências Humanas, v. 5, n. 1, 2015

OLSON, D. e BRUNER, J. Psicologia popular e pedagogia popular. In., N. Educação e desenvolvimento humano. Artmed - Porto Alegre: Artmed, 2000, p. 21-35.

SANTIAGO, Homero. Adorno, Auschwitz e a esperança na educação. Cadernos de Ética e Filosofia Política 6, São Paulo, USP, 1/2005, p. 111-122.

SAVIANI, D.A Filosofia da educação e o problema da inovação na educação, 1995.

SÉRGIO, Marina Carradore et al. Indicadores quantitativos de inovação como suporte ao processo de gestão de ideias. Revista E-Tech: Tecnologias para Competitividade Industrial, v. 8, n. 1, p. 69-86, 2015.

TEIXEIRA, Cláudia Maria Francisca. Inovar é preciso: concepções de inovação em educação dos programas Proinfo, Enlaces e Educar. 2010. Florianópolis.

WENTLING, Tim L. et al. E learning: a review of literature. Knowledge and Learning Systems Group, University of Illinois at Urbana-Champaign, 2000.. 
WILLIAMS, R. Cultura: Paz e Terra. São Paulo, 2008 